\title{
Use of Natalizumab in Multiple Sclerosis Patients
}

\author{
Paul W. O'Connor
}

Can. J. Neurol. Sci. 2010; 37: 98-104

Natalizumab is a monoclonal antibody indicated in Canada as monotherapy for the treatment of multiple sclerosis (MS) patients with relapsing-remitting disease. The phase III clinical trial of natalizumab monotherapy demonstrated substantial improvements in clinical and magnetic resonance imaging (MRI) parameters in patients with relapsing-remitting MS, with statistically significant reductions in annualized relapse rate (68\%), sustained disability progression (42-54\% over two years), and the number of gadolinium-enhancing lesions (92\%) and new or enlarging T2 lesions ( $83 \%$; all $\mathrm{p}<0.001$ compared to placebo). ${ }^{1}$ Despite the recognized limitations of measuring surrogate endpoints such as short-term disability progression and changes in lesion burden on MRI in MS trials of short duration (i.e., two years relative to the life-long disease) ${ }^{2,3}$ clinical experience to date generally supports the efficacy and tolerability profile of this agent as reported in clinical trials.

Natalizumab is generally recommended in MS patients who have had an inadequate response to, or are unable to tolerate, other therapies for MS. Although natalizumab has been available in Canada for more than two years, there remains uncertainty among community and academic neurologists on how best to utilize this drug in clinical practice. In light of rare reports of serious and potentially fatal infections (such as progressive multifocal leukoencephelopathy [PML]) and malignancies in MS patients exposed to natalizumab, ${ }^{1,4-6}$ it is paramount for clinicians to weigh the benefits of natalizumab therapy against the potential risks.

The following management recommendations relating to the use of natalizumab in patients with MS were developed by a national, independent expert panel that convened in February, 2009. The primary objective was to develop a clear and practical set of consensus-based ${ }^{i}$, non-binding recommendations on the use of natalizumab in MS patients, based on available evidence and the panel's experience to date. ii These recommendations discuss issues related to natalizumab treatment, patient selection, and usage, many of which have not yet been formally investigated and/or for which robust evidence is currently lacking to inform clinical practice.

\section{Patient Selection}

The standard use of natalizumab is in patients with clinically definite MS with failure or inadequate response to an adequate course (i.e., at least one year) of one or more conventional disease modifying drugs (DMDs), such as beta-interferons and glatiramer acetate. Failure or inadequate response was defined by this group as continued or increased relapse rate from pretreatment levels, and sustained worsening disability, with or without lesions on MRI. In other words, MRI changes alone, in the absence of clinical disease activity such as either relapses or disability progression, should not prompt a change in therapy.
The degree to which relapse rate and disability progression are unacceptable is a judgment call and depends on the patient's initial relapse rate and EDSS level. In general, the lower the initial EDSS score, the less significant an increase in disability is because it takes less increase in neurological disability to trigger an increase in the EDSS. Furthermore, the EDSS scale is not linear.

Importantly, use of natalizumab should occur when there is still evidence of ongoing central nervous system (CNS) inflammatory activity (i.e., relapses and/or enhancement on MRI).

In general, patients with an EDSS score that is chronically greater than 6.0 (i.e., requiring intermittent or unilateral constant assistance [cane, crutch or brace] required to walk about 100 metres with or without resting) are unlikely to have a predominantly inflammatory disease, and are less likely to benefit from natalizumab therapy. There is currently no evidence to suggest that natalizumab would be effective in patients with worsening due to primary or secondary progressive MS disease alone.

Other patient groups that could be considered for treatment with natalizumab include the following:

- Patients with clinically definite MS with intolerability to conventional DMDs (e.g., intolerable injection side effects, depression, needle phobia) and with evidence of inflammatory disease activity (e.g., clinical relapses with or without gadolinium enhancement [Gd+] on MRI).

- De novo treatment or as second-line therapy in patients with rapidly-evolving severe $\mathrm{MS}$, defined by this panel as $\geq 2$ disabling relapses (i.e., dysfunction that affects activities of daily living and is associated with incomplete recovery) within one year $\mathrm{OR} \geq 2$ moderate to severe relapses with complete recovery within one year AND an active MRI scan (defined as the presence of $\mathrm{Gd}+$ enhancing lesions or $\geq 2$ new $\mathrm{T} 2$ lesions).

\footnotetext{
i "Consensus" was defined as a majority view of prevailing practice or wisdom, without implying unanimity.

ii Individual panel members' experience with natalizumab at the time of the meeting was varied both in terms of total patient numbers and duration of treatment.
} 
- Patients younger than 18 years-of-age who meet the above criteria for treatment with natalizumab, although data are lacking in this patient population; notably, in younger patients of childbearing age, natalizumab might be preferable over conventional chemotherapeutic agents with fertility and/or teratogenic risks (e.g., mitoxantrone, cyclophosphamide, azathioprine).

- Older patients (>64 years-of-age) meeting the above criteria for treatment should be considered with added caution since cases of PML to date suggest that older patients may be at greater risk (see later section on PML).

\section{Pre-Treatment Tests And Procedures}

According to the Canadian product label, natalizumab is contraindicated in patients who are immmunocompromised due to immunosuppressant or antineoplastic therapies, or immunodeficiences (HIV, leukemias, lymphomas, etc.). ${ }^{7}$ Natalizumab is also contraindicated in patients who have or have had PML, or who are hypersensitive to the drug or any of the ingredients in the formulation or component of the container.

The following recommendations outline what constitutes an appropriate work-up before initiating treatment with natalizumab, as well as monitoring tests to carry out after a patient has started receiving natalizumab therapy.

Prior to initiating treatment, a patient's baseline immune competence should be assessed by the following tests; if any results are positive or abnormal, natalizumab should not be initiated until the cause of the immunocompromised status is known and has resolved:

- Clinical history with a focus on prior and current infections (i.e., prior infections that were unusual or severe as well as any current infections) and prior exposure to chemotherapy (see below for washout period recommendations).

- Complete blood count and differential.

- CD4 / CD8 counts should be strongly considered (levels should be $>500$ and $>250$ cells $/ \mu \mathrm{L}$, respectively $^{8}$ ).

- An HIV screen should be strongly considered (with the patient's consent); natalizumab is not recommended by this panel for use in HIV-positive individuals, even if they have normal CD4 / CD8 counts, at the least not in the absence of consultation between neurology, immunology and infectious disease specialists.

- A chest X-ray should be considered where indicated (e.g., patients coming from areas where tuberculosis is endemic, patients with cough or abnormalities on auscultation).

Other baseline tests that should be conducted prior to initiation of natalizumab include:

- Detailed medical history with full neurological exam and EDSS performed by a trained neurologist.

- General physical examination.

- Brain MRI at baseline unless one is available from the previous six months (a contrast enhanced study is recommended using a standardized MRI protocol, e.g., the Consortium of MS Centers protocol) $;{ }^{9}$ consider including T1, T2, proton density (PD), and fluid attenuated inversion recovery (FLAIR) imaging.

- Liver function tests.

- Patients with risk factors for malignancy should be evaluated on an individual basis.

- A cerebrospinal fluid (CSF) exam is optional and there is no specific need to do this. Storing CSF for future analysis in the unlikely event (1/1000) of PML should perhaps be considered in a research context but is not part of routine care.

- Although there is no evidence to suggest that Varicella poses a risk with this drug, it would not be unreasonable for the clinician to enquire about a patient's Varicella history and perform blood tests for antibodies against this infectious agent if a question arose.

- No specific vaccinations are recommended prior to starting natalizumab. The use of live or live-attenuated vaccines during therapy is not recommended; data on this question are not available however.

\section{Recommendations On Washout Periods}

At the time of writing these recommendations, there was little evidence to guide clinicians on adequate washout periods when transitioning patients from other DMDs to natalizumab. The decision on appropriate washout periods should be individualized, since a treatment-free period may not be desirable in all patients, particularly those with rapidly worsening disease and/or high disease activity.

\section{Interferon-beta $(\mathrm{IFN} \beta)$ or glatiramer acetate:}

There is no direct evidence to suggest that sequential use of these agents and natalizumab is associated with safety risks. Indeed, the product label suggests that a washout period is not necessary when switching from IFN $\beta$ or glatiramer acetate. A recent short-term study investigating the addition of natalizumab to glatiramer acetate suggests the combination is safe and well tolerated during six months of therapy. ${ }^{10}$ However, the SENTINEL trial suggests there might be a safety risk associated with simultaneous use of natalizumab and IFN $\beta .{ }^{11}$ In the absence of conclusive data, a washout period may not be absolutely necessary but if one is performed, the recommended duration is of up to four weeks when switching patients from IFN $\beta$ or glatiramer acetate to natalizumab.

\section{Chemotherapy}

The safety of treating patients previously exposed to chemotherapeutic agents remains a highly controversial topic. Patients who have previously received high-dose chemotherapy generally represent a patient population with high unmet needs, however, their risk for serious adverse events related to natalizumab therapy may also be greater. At the time this report was written, five of nine post-marketing cases of PML that occurred in patients who were exposed to natalizumab have had a history of treatment with chemotherapeutic agents that are known to suppress the immune system.

Transitioning patients from chemotherapeutic agents to natalizumab requires both the passage of time and normal CD4 and CD8 T-cell counts. Patients who have normal CD4 and CD8 counts can be considered ready for the initiation of natalizumab three or more months after the discontinuation of chemotherapy. For those patients who have previously received high-dose mitoxantrone or cyclophosphamide or prolonged treatment with azathioprine (i.e., $\geq 5$ years), a longer washout period of between three to six months is advised. These higher-risk patients should not be started on natalizumab unless there is evidence of adequate immune function (i.e., normal CD4 and CD8 and 
possibly B cell counts, as well as no recent history of opportunistic infections) and no sign of acute infection.

\section{Monitoring While On Natalizumab Treatment}

Patients should be evaluated at minimum every six months, and earlier if there are any concerns such as hypersensitivity reactions, infusion reactions, new neurological symptoms, or disease worsening. Reports from infusion centres can be helpful in identifying patients who should be contacted for an earlier consultation with the treating neurologist.

The following evaluations should form part of routine follow-up visits:

- History of any new neurological symptoms and complete neurological exam.

- Update history of general health with a physical exam looking particularly for signs of opportunistic infection, malignancy (skin or otherwise) and liver disease.

- $\mathrm{CBC}$ and differential (to screen for evidence of immunosuppression with leucopenia).

- LFTs in patients at risk for hepatotoxicity (e.g., patients with a history of liver disease, alcohol abuse, etc.) since some cases of hepatotoxicity have been reported in patients with pre-existing liver disease or in the presence of other drugs that have been associated with hepatic injury. In some patients, liver injury recurred upon rechallenge.

Notably, clinicians should actively evaluate patients for any new neurological symptoms or atypical patterns of disease that might be signs of PML. For example, visual field deficits, progressive hemiparesis, cognitive dysfunction, and other cortical signs including aphasia, have been observed in MS patients who have developed PML (see Table 1).

A new MRI of the brain should be obtained annually. This can serve as a new baseline against which comparisons can be made should PML be suspected in an individual patient. Imaging can also serve (in part) to assess the patient's response to natalizumab therapy.

\section{Managing Potential Adverse Events}

In general, natalizumab has been associated with a favourable tolerability profile, with few treatment-emergent adverse events. ${ }^{1}$

Headache is one of the most common adverse events, and can be treated symptomatically with over-the-counter analgesics such as acetaminophen or ibuprofen.

Transient, mild cutaneous rashes and/or flushing have occurred in rare cases and generally disappear spontaneously. Since these reactions can be distressing to patients, mild rashes and flushing can be treated pre-emptively by the administration of antihistamines and/or cortisone prior to infusion of natalizumab.

In rare cases, infusion-related allergic hives (urticaria) or other hypersensitivity reactions may occur. This adverse event is an indication to stop infusion of the drug and to permanently discontinue natalizumab treatment. Hypersensitivity reactions have generally occurred during the second infusion, and are often associated with the presence of neutralizing antibodies (NAbs). ${ }^{1}$ More severe hypersensitivity reactions include shortness of breath, tachycardia and hypotension.
Patients may complain of fatigue in the days prior to and following the first few natalizumab infusions; this adverse event is generally self-limiting and does not require treatment. Notably, several experts on the panel noted that some patients report improvements in fatigue when switching from other DMDs to natalizumab.

No special treatment is recommended or required for patients with a history of recurrent herpes simplex. At this time, it is recommended that herpes simplex infections during natalizumab therapy be managed symptomatically including the use of valacyclovir.

Serious adverse events should be reported to Health Canada's Vigilance Program at the National Adverse Event Reporting Centre (toll-free at 866-234-2345 or by email to Canada Vigilance@hc-sc.gc.ca). Furthermore, this panel recommends that the reporting of PML be mandatory.

\section{Possible Considerations For Withholding Natalizumab Therapy}

Based on the theoretical risks associated with an immunocompromised status, natalizumab administration should be withheld in patients with active, acute infections until the infection resolves. In the case of viral colds or influenza, infusions can be administered in the absence of fever. For patients with febrile cold or flu infections, infusions should be withheld until the fever resolves. Likewise, patients should not be infused with natalizumab until an episode of shingles has resolved.

Natalizumab dosing should be suspended in patients with new neurological symptoms suggestive of PML (see later section on identification and management of possible PML cases).

Reports from an ongoing pregnancy registry suggest that teratogenicity has not occurred when female patients receiving natalizumab have become pregnant. Although there are no evidence-based data available to guide clinical practice on this issue, it is advised that patients planning a pregnancy should discontinue natalizumab three months prior to conceiving. Similarly, women should not breastfeed their infants while receiving natalizumab since it is not known whether this agent crosses into breast milk.

A mother's risk/benefit profile should be reassessed shortly after delivery, and if she is at significant risk of relapse, she should be advised to re-start natalizumab therapy.

\section{Possible Considerations For Discontinuation Of Therapy}

The development of a bona fide hypersensitivity reaction (e.g., hives) is an indication to immediately and permanently discontinue natalizumab therapy, as discussed in a previous section of these recommendations.

Loss or lack of efficacy, defined as the failure of natalizumab to mitigate the pre-treatment relapse pattern after a minimum of one year of therapy, constitutes grounds for considering discontinuation. Confirmed EDSS progression alone should not necessarily be sufficient justification for discontinuing therapy, nor should increased MRI activity in a clinically stable patient. Nevertheless, progressively worsening disease observed on two consecutive visits and/or worsening MRI activity can be considered indicators for loss or lack of efficacy, and should be closely monitored. 


\section{Neutralizing antibodies}

As with other protein therapies, exposure to natalizumab has been associated with the development of NAbs, which significantly diminish clinical benefits of therapy. In the phase III clinical studies of natalizumab, approximately $6 \%$ of patients developed 'persistently positive' (defined as more than one positive result) antinatalizumab antibodies. ${ }^{1,11}$ Evidence suggests that NAbs most commonly appear in the first six months of therapy, and that transiently positive NAbs disappear within this time frame.

All patients should therefore be tested for the presence of NAbs six months after initiation of natalizumab therapy. In the event that a positive result is obtained, the NAbs test should be repeated as soon as possible; natalizumab infusions should be suspended until the results of the second NAbs test are available. Natalizumab should be permanently discontinued in patients with persistently positive NAbs.

Since NAbs generally do not develop after six months of therapy, there is no indication for routine periodic NAbs testing beyond this timeframe, unless indicated (e.g., development of hives or radiologic evidence of loss of response).

The drug manufacturer arranges for reimbursed third-party NAbs testing; protocols for sample acquisition and shipping can be obtained directly from the manufacturer.

\section{Rebound of disease activity}

Evidence suggests that natalizumab provides beneficial effects shortly after initiation of therapy, but that it does not induce long-lasting remissions after drug discontinuation. ${ }^{1}$ Therefore, continuous treatment is required for the maintenance of clinical benefits. The suspension of natalizumab in 2005 due to the occurrence of two cases of PML in MS patients during clinical trials provides important information on patient outcomes following discontinuation of natalizumab therapy. Patients in the AFFIRM study who discontinued natalizumab therapy had a return of clinical and MRI disease activity to onstudy placebo levels within four months. ${ }^{12}$

A natalizumab re-dosing study in patients enrolled in the AFFIRM study suggests that patients who discontinue treatment after only two infusions and are then re-infused at a later time, are more likely to develop infusion reactions and NAbs. ${ }^{13}$ Furthermore, these patients appear to be more susceptible to a rebound in $\mathrm{T} 2$ disease activity upon discontinuation after receiving only a few infusions. ${ }^{14}$ Therefore, clinicians should avoid re-starting natalizumab in patients who discontinued or interrupted treatment shortly after initiation of therapy.

\section{Identification And Management Of Possible PML Cases}

At the time of final writing of these recommendations, there were twenty-seven cases of PML in patients exposed to natalizumab monotherapy worldwide, in addition to the three cases reported during clinical development (of which two MS patients received combination natalizumab and IFN $\beta$ therapy and a third patient was being treated for Crohn's disease).$^{4-6}$

Older age and male gender might be risks for developing PML. Chemotherapy exposure and increasing duration of treatment are established risks. Clinicians should use natalizumab with particular caution in older patients with prior exposure to chemotherapy and increasing duration of treatment, particularly after two years.

Patient education is a critical component of vigilance for PML. Prior to initiating therapy with natalizumab, patients should be informed of the risks of PML and be provided with written educational materials about the drug, its potential side effects, how to recognize signs and symptoms of PML, and what to do if new neurological symptoms develop.

\section{Identification of possible PML cases}

All patients should be instructed to promptly report any new neurological symptoms to their clinician; reminders should be given to patients at each clinical visit. Tables 1 and 2 outline clinical and MRI features that help to distinguish PML from MS relapses.

An algorithm for evaluating possible PML cases has been

Table 1: Clinical signs and symptoms of typical MS and PML ${ }^{15}$

\begin{tabular}{|c|c|c|}
\hline & MS & PML \\
\hline Onset & Acute & Subacute \\
\hline Evolution & $\begin{array}{ll}- & \text { Over hours to days } \\
\text { - } & \text { Normally stabilize } \\
- & \text { Resolve spontaneously } \\
& \text { or with treatment } \\
\end{array}$ & $\begin{array}{ll}\text { - } & \text { Over weeks } \\
\text { - } & \text { Progressive }\end{array}$ \\
\hline $\begin{array}{l}\text { Clinical } \\
\text { presentation }\end{array}$ & $\begin{array}{ll}\text { - } & \text { Diplopia } \\
\text { - } & \text { Paresthesia } \\
\text { - } & \text { Optic neuritis } \\
\text { - } & \text { Myelopathy } \\
\text { - } & \text { Ataxia } \\
\end{array}$ & $\begin{array}{ll}\text { - } & \text { Cortical symptoms / signs } \\
\text { - } & \text { Behavioural and paraparesis } \\
\text { - } & \text { Neuropsychological alteration } \\
\text { - } & \text { Retrochiasmal visual deficits } \\
\text { - } & \text { Hemiparesis }\end{array}$ \\
\hline
\end{tabular}

proposed by Kappos et al and endorsed by the drug manufacturer (Figure) ${ }^{7,15}$ If there is a suspicion of PML, dosing should be suspended, an MRI with Gd+ enhancement should be obtained as soon as possible, and the drug manufacturer should be contacted. If the MRI shows features suggestive of PML, a cerebrospinal fluid sample should be obtained and tested using the polymerase chain reaction for the presence of JC virus DNA, the causative agent of PML. Natalizumab re-dosing should only be initiated if the diagnosis of PML is excluded and the continuation of treatment is deemed by the clinician to be appropriate in an individual patient.

\section{Management of confirmed PML cases}

The development of PML can be fatal; however, if cases are identified and treated at an early stage, the prognosis may be more favourable. At this time, it is recommended that dosing continue to be suspended in patients with confirmed PML and that immunoadsorption or plasma exchange (PLEX) therapy be instituted to rapidly remove natalizumab from plasma and to restore normal leukocyte function. ${ }^{16}$

Multiple sclerosis patients who develop PML are at risk of developing the immune reconstitution inflammatory syndrome 


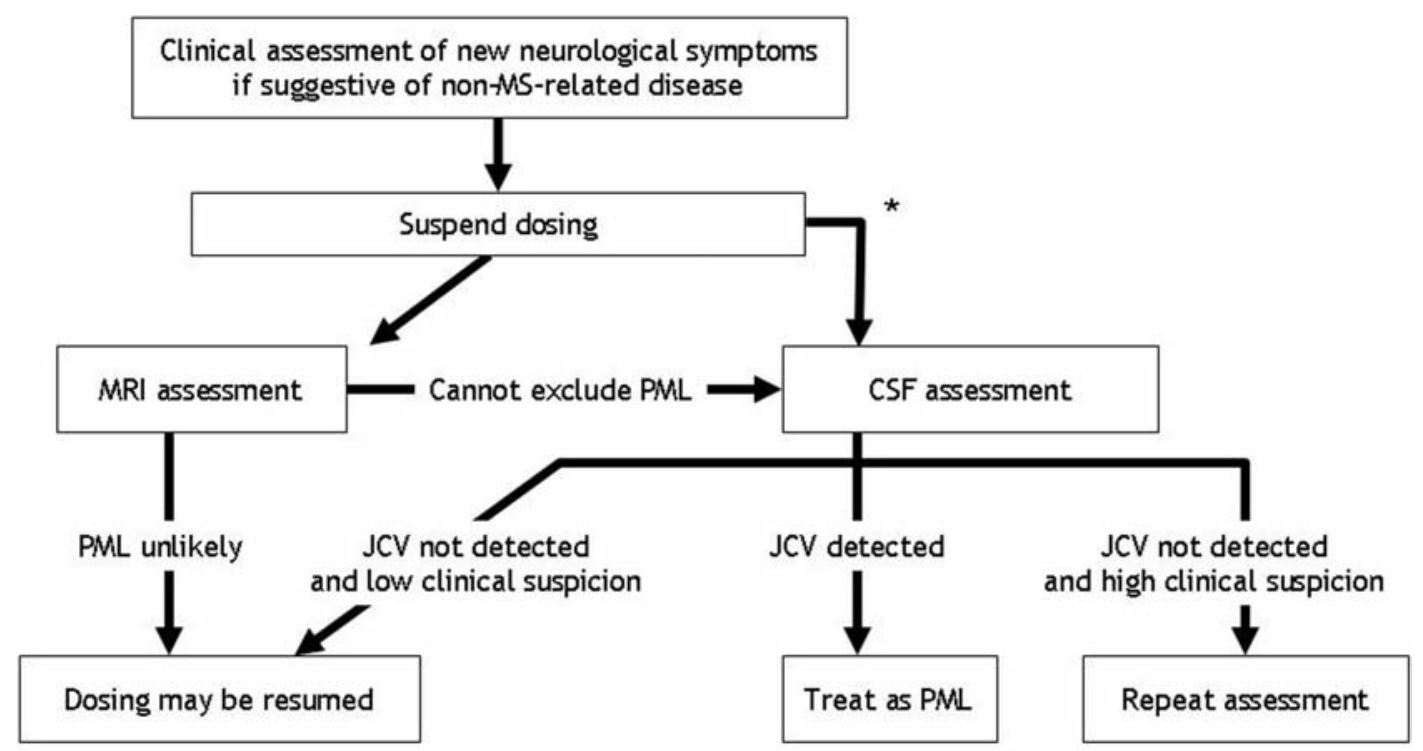

Figure: Suggested diagnostic algorithm for natalizumab-treated MS patients with new or worsening neurological symptoms. Reproduced with permission from Kappos et al ${ }^{15}$ and Tysabri ${ }^{\circledR}$ Product Monograph. ${ }^{7}$ MS: multiple sclerosis; CSF: cerebrospinal fluid; PML: progressive multifocal leukoencephalopathy; MRI: magnetic resonance imaging; JCV: JC virus. * If PML is suspected on the basis of clinical presentation and MRI is not readily available, CSF assessment to exclude PML should be considered before MRI.

(IRIS) within one to three months of the discontinuation of natalizumab. Importantly, immunoadsorption and PLEX may increase the probability and hasten the onset of IRIS. Although IRIS appears to be a desirable outcome insofar as it heralds the recovery of the immune system, it can potentially be fatal. Patients developing IRIS should be treated with intravenous steroids.

\section{Other Opportunistic Infections And Malignancies}

Although rare, other opportunistic infections may also occur in patients exposed to natalizumab. There have been no statistically significant differences in incidence rates of opportunistic infections and malignancies in patients treated with natalizumab compared to placebo in randomized trials. ${ }^{1,11}$ The incidence of infections and malignancies in the post-marketing setting is being actively monitored in long-term registry trials of natalizumab therapy. To date, there have been no unexpected serious adverse events and the incidence of serious adverse events including infections and hypersensitivity reactions has been similar to that observed in clinical trials. ${ }^{17}$ However, the potential for serious complications to emerge as larger numbers of patients are exposed for longer periods of time exists and must continue to be monitored. ${ }^{18}$

Malignancy has been a concern with some other monoclonal antibody therapies; although there were no observed differences in incidence rates or the nature of malignancies between natalizumab- and placebo-treated patients in clinical trials, ${ }^{1,11}$ clinicians should be vigilant in monitoring patients for signs and symptoms of malignancy.

\section{Vaccinations While On Treatment}

According to the drug manufacturer, there are no data available on the effects of vaccination in patients receiving natalizumab. ${ }^{7}$ Likewise, there are no data to suggest that infections can be transmitted via live or live-attenuated vaccines administered to patients receiving natalizumab. Therefore, this group of experts recommends that live or live-attenuated vaccines should not be administered to patients receiving natalizumab. Patients requiring live or live-attenuated vaccination(s) should receive these prior to initiation of natalizumab therapy, wherever possible.

Despite the lack of evidence to guide clinicians at this time, there appears to be no reason to believe that killed vaccines would be ineffective in patients receiving natalizumab.

\section{Recommended Duration Of Natalizumab Therapy}

The optimal duration of natalizumab therapy has yet to be elucidated, and remains a controversial issue. The risk of PML increases with increasing duration of therapy and efforts to change the prescribing label in the USA were underway in late 2009. Other concerns include the uncertain duration of clinical and immune effects of natalizumab and the as-yet undefined susceptibility factors for PML and other opportunistic infections. Robust data on the risk of PML after three years of therapy have not yet been obtained.

At this time, this panel of experts does not endorse routine 'drug holidays' (e.g., utilization of natalizumab as an intermittent therapy) in patients who are demonstrating beneficial effects of therapy. Until more evidence is available, it is advised that 
Table 2: Lesion characteristics typical of MS and PML ${ }^{19}$

\begin{tabular}{|c|c|c|}
\hline Feature & MS & PML \\
\hline $\begin{array}{l}\text { Location of new } \\
\text { lesions }\end{array}$ & $\begin{array}{l}\text { Mostly focal; may affect entire brain and spinal } \\
\text { cord, in white and possibly grey matter; posterior } \\
\text { fossa lesions are rarely seen. }\end{array}$ & $\begin{array}{l}\text { Diffuse (i.e., large, extensive) lesions, mainly subcortical and } \\
\text { rarely periventricular, located almost exclusively in white } \\
\text { matter, although occasional extension to grey matter has been } \\
\text { seen; posterior fossa frequently involved (cerebellum). }\end{array}$ \\
\hline Borders & $\begin{array}{l}\text { Sharp edges; mostly round or finger-like in shape } \\
\text { (especially periventricular lesions), confluent with } \\
\text { other lesions; U-fibres may be involved. }\end{array}$ & $\begin{array}{l}\text { Ill-defined edges; infiltrating, irregular in shape, confined to } \\
\text { white matter, sparing grey matter, pushing against the cerebral } \\
\text { cortex; U-fibres destroyed. }\end{array}$ \\
\hline Mode of extension & $\begin{array}{l}\text { Initially focal; lesions enlarge within days or } \\
\text { weeks and later decrease in size within months. }\end{array}$ & $\begin{array}{l}\text { Lesions are diffuse and asymmetric, extending } \\
\text { homogeneously; no confluence with other lesions; confined to } \\
\text { white matter tracks, sparing the cortex; continuous } \\
\text { progression. }\end{array}$ \\
\hline Mass effect & Acute lesions show some mass effect. & $\begin{array}{l}\text { No mass effect even in large lesions (but lesion slightly abuts } \\
\text { cerebral cortex). }\end{array}$ \\
\hline $\begin{array}{l}\text { On T2-weighted } \\
\text { sequence }\end{array}$ & $\begin{array}{l}\text { Acute lesions: hyperintense centre, isointense } \\
\text { ring, discrete hyperintensity outside the ring } \\
\text { structure. } \\
\text { - Subacute and chronic lesions: hyperintense } \\
\text { with no ring structure. }\end{array}$ & $\begin{array}{l}\text { Diffuse hyperintensity, slightly increased intensity of newly } \\
\text { involved areas compared with old areas; little irregular signal } \\
\text { intensity of lesions. }\end{array}$ \\
\hline $\begin{array}{l}\text { On T1-weighted } \\
\text { sequence }\end{array}$ & $\begin{array}{l}\text { Acute lesions: densely hypointense (large lesions) } \\
\text { or isointense (small lesions); increasing signal } \\
\text { intensity over time in } 80 \% \text {; decreasing signal } \\
\text { intensity (axonal loss) in about } 20 \% \text {. }\end{array}$ & $\begin{array}{l}\text { Slightly hypointense at onset, with signal intensity } \\
\text { decreasing over time and along the affected area; no reversion } \\
\text { of signal intensity. }\end{array}$ \\
\hline $\begin{array}{l}\text { On FLAIR } \\
\text { sequence }\end{array}$ & Hyperintense, sharply delineated. & $\begin{array}{l}\text { Hyperintensity more obvious; true extension of abnormality } \\
\text { more clearly visible than in T2-weighted images. }\end{array}$ \\
\hline With enhancement & $\begin{array}{l}\text { - Acute lesions: dense homogeneous } \\
\text { enhancement, sharp edges. } \\
\text { - Subacute lesions: ring enhancement. } \\
\text { - Chronic lesions: no enhancement. }\end{array}$ & $\begin{array}{l}\text { Usually no enhancement, even in large lesions; in patients } \\
\text { with HIV, some peripheral enhancement is possible, } \\
\text { especially under therapy. }\end{array}$ \\
\hline Atrophy & $\begin{array}{l}\text { Focal atrophy possible due to focal white matter } \\
\text { degeneration; no progression. }\end{array}$ & No focal atrophy \\
\hline
\end{tabular}

Adapted from Yousry et al ${ }^{19}$

clinicians re-assess an individual patient's risk/benefit profile on an annual basis; decisions on continuation of natalizumab therapy should be informed by the most recent incidence rates of PML and other serious adverse events, and the patient's response to therapy. Notably, patients not benefiting from natalizumab after a trial of one year (for example, patients with increased clinical disease activity from pre-treatment levels) should not continue to receive this therapy.

\section{Conclusions}

Evidence to date supports the efficacy of natalizumab in patients with MS who have signs of ongoing inflammation (e.g., clinical relapses with or without new MRI lesions). Although this agent has a favourable tolerability profile, its associated risk with PML warrants careful patient selection, ongoing monitoring and clinical vigilance after institution of therapy. There remain unanswered questions regarding optimal use of natalizumab that warrant investigation in prospective, controlled trials. Until such studies are completed, clinical practice will continue to evolve and be guided by available evidence and growing practical experience with this agent. At this time, it is recommended that patients treated with natalizumab be:

1. Carefully selected based on an individualized benefit-risk assessment

2. Vigilantly monitored while on natalizumab therapy to proactively address any safety issues should they occur

3. Routinely assessed for continued evidence of benefit of therapy with treatment discontinued in the absence of clinical benefits

4. Enrolled in long-term safety registries as available (e.g., Tysabri Observational Program [TOP] or Tysabri Global Observational Program in Safety [TYGRIS])

These consensus-based recommendations were prepared by Paul O'Connor with input and approval from the panel of Canadian MS neurologists experienced with natalizumab therapy, and these individuals are co-signers of this clinical commentary article (listed alphabetically): Bhan V, Devonshire 
V, Duquette P, Girard M, Gomori A, Hashimoto S, Jacques F, Kremenchutzky M, Lapierre Y, Lee L, Paulseth J, Selchen D, Stewart B.

\section{DeClaration}

Paul O'Connor has received consulting fees and/or research support for MS trials from Abbott, Bayer, Biogen Idec, BioMS, Cognosci, Daiichi Sankyo, EMD Serono, Genentech, Genmab, Novartis, Roche, Sanofi Aventis, and Teva.

\section{REFERENCES}

1. Polman CH, O'Connor PW, Havrdova E, Hutchinson M, Kappos L, Miller DH, et al. A randomized placebo-controlled trial of natalizumab for relapsing multiple sclerosis. N Engl J Med. 2006 Mar 2;354:899-910.

2. Marrie R. You get what you measure. Evaluating endpoints in MS clinical trials. Neurology. 2008 Aug 26;71:620-1.

3. Koch-Henriksen N. No shortcuts to outcome in MS clinical trials? Neurology. 2009 Feb 24;72:686-7.

4. Langer-Gould A, Atlas SW, Green AJ, Bollen AW, Pelletier D. Progressive multifocal leukoencephalopathy in a patient treated with natalizumab. N Engl J Med. 2005 Jul 28;353(4):375-81. Epub 2005 Jun 9.

5. Kleinschmidt-DeMasters BK, Tyler KL. Progressive multifocal leukoencephalopathy complicating treatment with natalizumab and interferon-beta-1a for multiple sclerosis. N Engl J Med. 2005 Jul 28;353(4):369-74. Epub 2005 Jun 9.

6. Van Assche G, Van Ranst M, Sciot R, Dubois B, Vermeire S, Noman M, et al. Progressive multifocal leukoencephalopathy after natalizumab therapy for Crohn's disease. N Engl J Med. 2005 Jul 28;353(4):362-8. Epub 2005 Jun 9.

7. Biogen-Idec Canada Inc. Tysabri (natalizumab) Product Monograph. January 9, 2009.

8. Gold R, Jawad A, Miller DH, Henderson DC, Fassas A, Fierz W, et al. Expert opinion: guidelines for the use of natalizumab in multiple sclerosis patients previously treated with immunomodulating therapies. J Neuroimmunol. 2007 Jul;187: 156-8. Epub 2007 May 11.

9. Consortium of MS Centers. Consortium of MS Centers MRI protocol for the diagnosis and follow-up of MS. [cited 2009 May] Available from: http://www.mscare.org/cmsc/images/pdf/ mriprotocol2009.pdf.

10. Goodman AD, Rossman H, Bar-Or A, Miller A, Miller DH, Schmierer $\mathrm{K}$, et al. for the GLANCE Investigators. GLANCE: results of a phase 2, randomized, double-blind, placebocontrolled study. Neurology. 2009 Mar 3;72(9):806-12.
11. Rudick RA, Stuart WH, Calabresi PA, Confavreux C, Galetta SL, Radue EW, et al. Natalizumab plus interferon beta-1a for relapsing multiple sclerosis. N Engl J Med. 2006 Mar 2;354: 911-23.

12. O'Connor PW, Goodman A, Kappos L, et al. Results of clinical and magnetic resonance imaging analyses following cessation of natalizumab dosing in patients with multiple sclerosis. Presented at the European Committee for Treatment and Research in Multiple Sclerosis Annual Congress, 29 September 2006; Madrid, Spain.

13. O'Connor P, Goodman A, Kappos L, et al. Safety of natalizumab upon re-dosing: preliminary results from STRATA study. Presented at the European Committee for Treatment and Research in Multiple Sclerosis Annual Congress, 11-14 October, 2007; Prague, Czech Republic.

14. Vellinga MM, Casteligns JA, Barkhof F, Uitdehaag BM, Polman $\mathrm{CH}$. Postwithdrawal rebound increase in T2 lesional activity in natalizumab-treated MS patients. Neurology. 2008 Mar 25;70(13 Pt 2):1150-1. Epub 2007 Sep 13.

15. Kappos L, Bates D, Hartung HP, Havrdova E, Miller D, Polman $\mathrm{CH}$, et al. Natalizumab treatment for multiple sclerosis: recommendations for patient selection and monitoring. Lancet Neurol. 2007 May;6:431-41.

16. Khatri BO, Man S, Giovannoni G, Koo AP, Lee JC, Tucky B, et al. Effect of plasma exchange in accelerating natalizumab clearance and restoring leukocyte function. Neurology. 2009 Feb 3;72: 402-9.

17. Panzara MA, Baldinetti F, Belcher G, Bozic C, Hyde R, Kim R, et al. Natalizumab utilization and safety in patients with relapsing MS: Updated results from TOUCH ${ }^{\mathrm{TM}}$ and TYGRIS. Poster presented at the European Committee for Treatment and Research in Multiple Sclerosis Annual Congress, 17-20 September 2008, Montreal, Canada (Poster P488).

18. Goodin DS, Cohen BA, O'Connor P, Kappos L, Stevens JC; Therapeutics and Technology Assessment Subcommittee of the American Academy of Neurology. Assessment: The use of natalizumab (Tysabri) for the treatment of multiple sclerosis (an evidence-based review). Report of the Therapeutics and Technology Assessment Subcommittee of the American Academy of Neurology. Neurology. 2008 Sep 2;71:766-73.

19. Yousry TA, Major EO, Ryschkewitsch C, Fahle G, Fischer S, Hou $\mathrm{J}$, et al. Evaluation of patients treated with natalizumab for progressive multifocal leukoencephalopathy. N Engl J Med. 2006 Mar 2;354(9):924-33. 